\title{
Photometry of OV Bootis at the Early Stage of 2017 Outburst
}

\author{
Kenji Tanabe*t \\ Okayama Univ.Sci. \\ E-mail: tanabe@big.ous.ac.jp \\ Hidehiko Akazawa \\ Okayama Univ.Sci. \\ E-mail: akazawa-hide@mx1.tamatele.ne.jp \\ Naoya Fukuda \\ Okayama Univ.Sci. \\ E-mail: fukudany@big.ous.ac.jp...
}

\begin{abstract}
We report our photometric observational results during the early stage of 2017 outburst of an ultra short orbital period cataclysmic variable star OV Bootis. From the obtained light curve, its period of the hump-like modulation is quite close to its orbital one This result suggests us that this variable star is thought to be neither WZ Sge-type nor SU UMa-type dwarf nova, taking into account the absence of rebrightening.
\end{abstract}

The Golden Age of Cataclysmic Variables and Related Objects IV 11-16 September, 2017

Palermo, Italy

*Speaker.

${ }^{\dagger}$ Another affiliation:Riken, Nishina Center for Accelerator-based Science 


\section{Introduction}

Cataclysmic variable star OV Bootis was first detected as one of the 32 emission line CVs by the systematic survey of SDSS (Sloan Digital Sky Survey) in 2003. So this object was at first named as SDSS J1507+52. However the previous outburst was recorded in 1984 in the photographic plate (H. Bengtsson).In 2005,Szkody et al. investigated these new $32 \mathrm{CVs}$ including this SDSS J1507.As a result they showed that this is an extremely short orbital period eclipsing dwarf nova (approximately $67 \mathrm{~min}$ ). This value is thought to be below the period minimum (78 $\mathrm{min}$ ) from the standard theory of CV evolution(see e.g. the textbook by Hellier(2001);in the Chapter 4.) From this point of view, this is an exotic CV. By performing high speed three-color photometry,Littlefair (2007) succeeded in revealing its detail of the binary structure. Uthas et al.(2011,see also PhD thesis of her) investigated this object using HST and concluded to be a possible halo object. The 2017 outburst is a good and lucky chance to investigate this exotic CV.We present our photometric observational results in the following sections.

\section{Detection of 2017 outburst.}

The 2017 outburst of OV Boo was detected on March 14.63th UT by M.Mukai,who had been looking for DNe outburst according to the list made by S.Yoshida,the leader of "MISAO Project" (image data analysis team). Soon after the detection, H.Akazawa (our collaborator and coauthor of this paper)started CCD time-series photometry from 14.76UT . This report is based on the results by a single observer and a $20 \mathrm{~cm}$ telescope with Rc filter CCD. Accordingly the photometric precision of the data are more definite than by multiple observers.

\section{Observation}

Observational Journal is given in Table 1.The observational system is as follows:

Observer :Hidehiko Akazawa, a skilled amateur observational astronomer

Telescope : $20 \mathrm{~cm}$ Meade (Schmidt Cassegrain type)

Filter: Rc

CCD :Moravian G2-1600.

The first night ,14.76th(UT) March 2017, is probably the earliest date of observation all over the world. Data processing software used was AIP4Win ver.2. Period of hump-shaped modulation is obtained by using PDM (Phase Dispersion Minimization;Stellingwerf(1978)).In the last column of the Table 1, the obtained periods $\theta$ (day) are given.

Overall light curve is given in Fig.1 


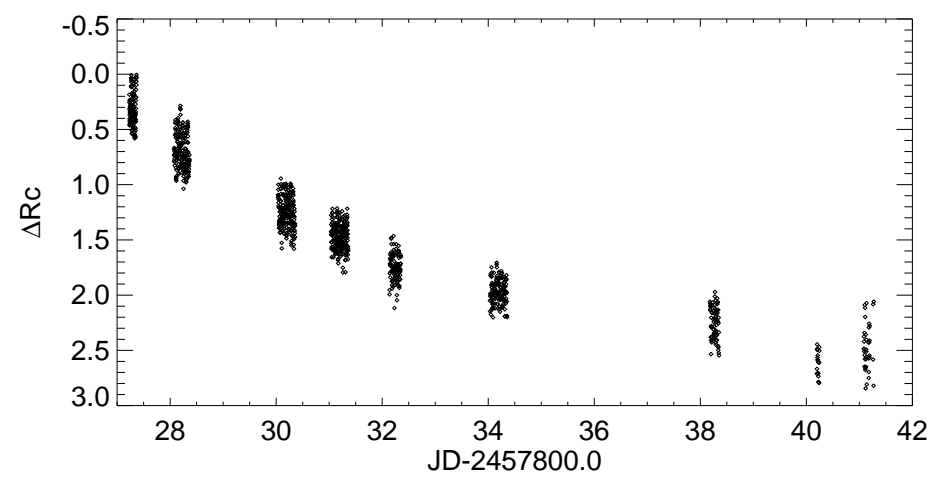

Figure 1: Overall light curve during the earliest stage of 2017 outburst.

Table 1.Journal of the observation.

\begin{tabular}{lcccccc}
\hline Date(UT) & $\begin{array}{c}\text { Start(JD) } \\
+24547800.0\end{array}$ & $\begin{array}{c}\text { End(JD) } \\
+24547800.0\end{array}$ & Exposure(sec) & Number & $\mathrm{D}(\mathrm{cm})$ & $\theta$ (day) \\
\hline 14/March & 27.22999 & 27.36664 & 90 & 127 & $20 \mathrm{~cm}$ & 0.0462 \\
15/March & 28.07363 & 28.36378 & 120 & 203 & $20 \mathrm{~cm}$ & 0.0459 \\
17/March & 30.03679 & 30.35659 & 120 & 224 & $20 \mathrm{~cm}$ & - \\
18/March & 31.03488 & 31.35771 & 120 & 228 & $20 \mathrm{~cm}$ & 0.0464 \\
19/March & 32.13840 & 32.35218 & 180 & 102 & $20 \mathrm{~cm}$ & 0.0464 \\
21/March & 34.13840 & 34.35495 & 180 & 153 & $20 \mathrm{~cm}$ & 0.0462 \\
25/March & 38.18257 & 38.35186 & 180 & 75 & $20 \mathrm{~cm}$ & 0.0464 \\
27/March & 40.19713 & 40.24581 & 180 & 24 & $20 \mathrm{~cm}$ & - \\
28/March & 41.08435 & 41.27145 & 180 & 41 & $20 \mathrm{~cm}$ & - \\
\hline
\end{tabular}

\section{Analysis of the Light curve}

Two examples of the light curve and associated $\theta$ (day),calculated period of the hump-like modulation are given in Fig.2 and 3. Temporal (daily) change of the light curve during the early stage of the outburst is given in Fig.4 The resultant averaged period of hump-like modulation is $66.9 \mathrm{~min}$. At the earliest stage dual hump-shaped structure can be seen. At its later stage deep eclipse can be seen. As a tentative ephemeris, we put $E=2457828.19314+0.04616 n$ However ,taking into account the time resolution of the present observation, it is better to express as follows:

$$
E=2457828.19+0.046 n \cdot(n=1,2,3, \cdots)
$$

Daily change of the light curve ,using the period 0.046 day, is given in Fig.4.Here we can see rather complicated feature at its earlier stage.Using the above formula,the obtained O-C diagram (Fig.5) shows that this period gives its linear change and no sign of period increase or decrease .Therefore this hump-shaped period seems to be identical with the orbital period obtained by Szkody et al.(2005) 

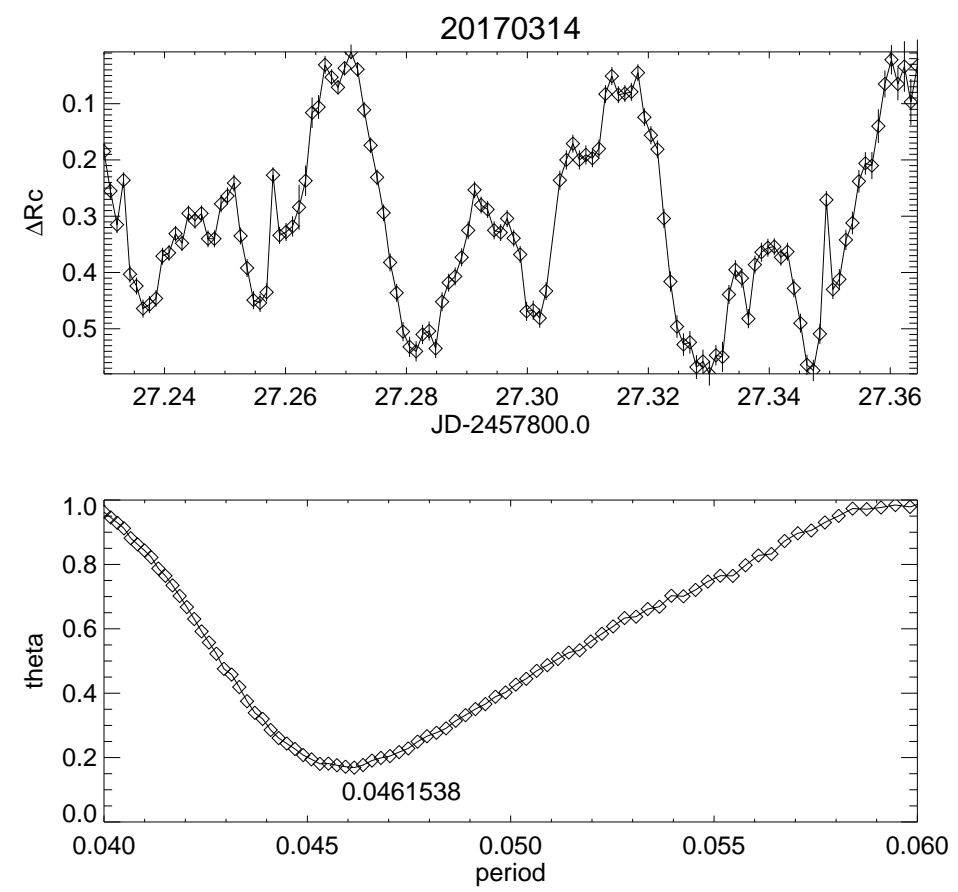

Figure 2: Representative light curve and the associated $\theta$ diagram:14th of March .
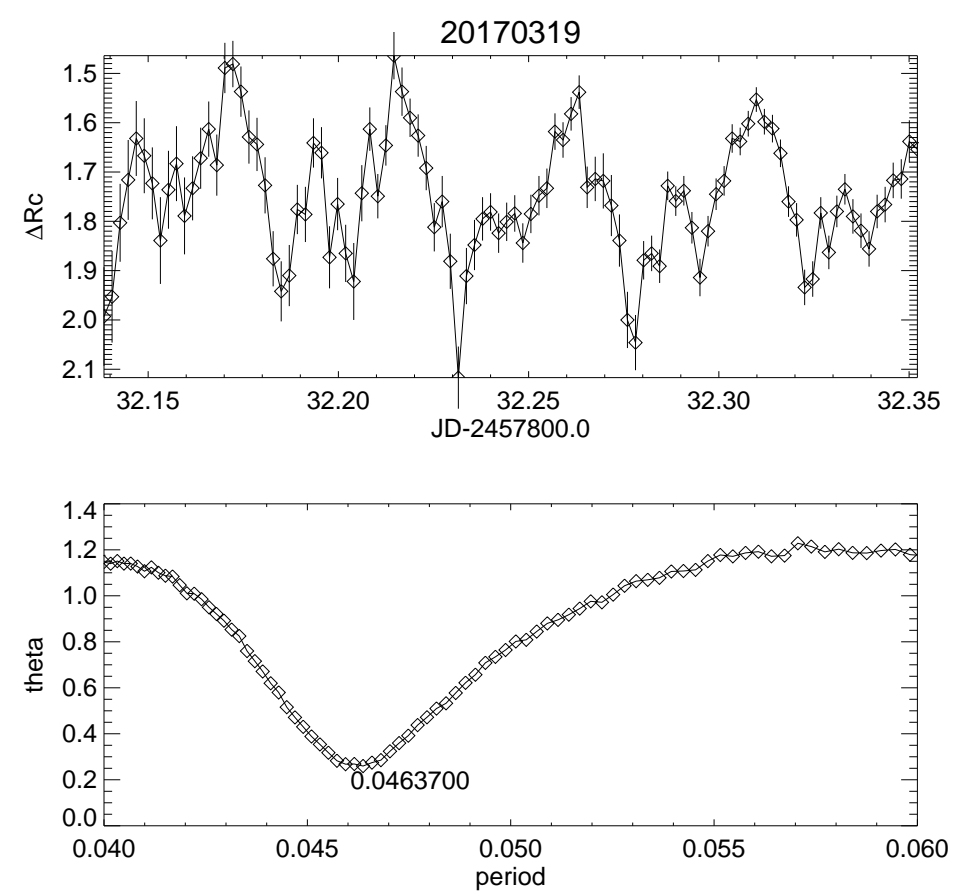

Figure 3: Representative light curve and the associated $\theta$ diagram:19th of March . 


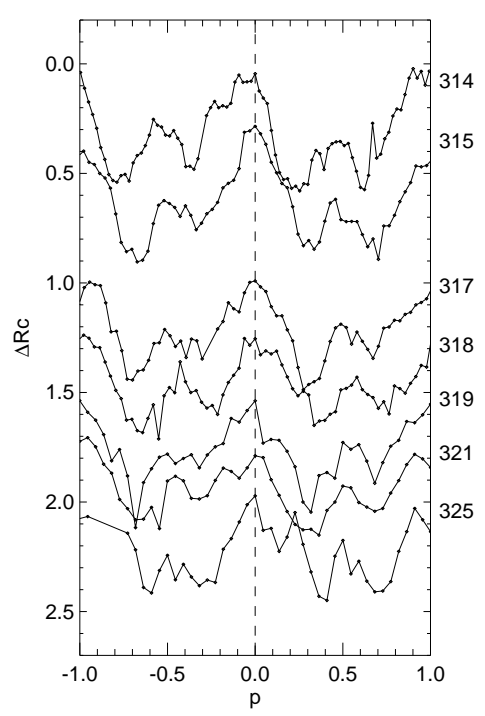

Figure 4: Temporal(daily) change of the light curve during the early stage of outburst. Abscissa is the phase,corresponding 0.046 day

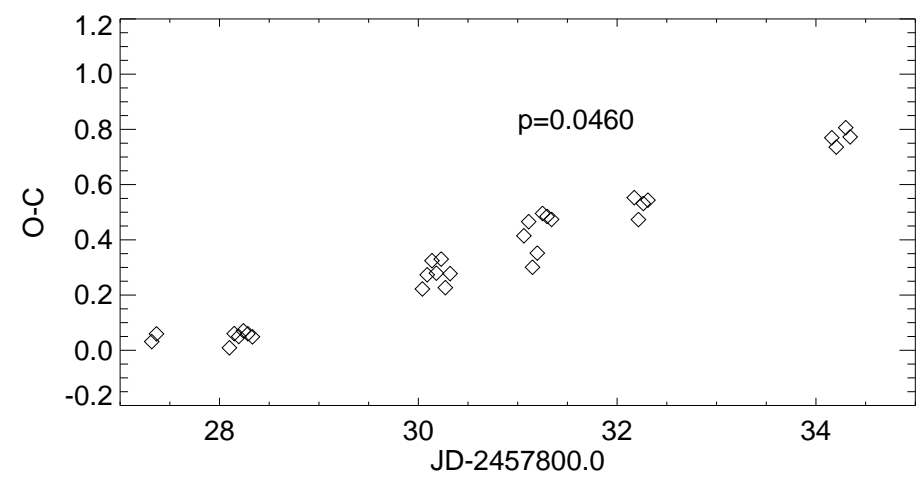

Figure 5: O-C diagram of 2017 outburst.

\section{Concluding Remarks.}

In spite of bright ( $\simeq 8$ magnitude) outburst, and long time quiescence ( $\sim 33$ year), outburst behavior of OV Bootis is very different from WZ Sge-type or SU UMa-type dwarf nova.The reason is as follows:

1. Period of the hump-like modulation is identical with its orbital period obtained from eclipse.

2. No rebrightening is detected by the end of August 2017.

3. At the early stage of 2017 outburst, dual hump-shaped feature can be seen(due to multiple bright spot ?).

At any rate ,this is an exotic dwarf nova . 


\section{Additional note}

Obtained period by PDM for entire data of the early stage of the outburst is 0.04615 day.

\section{References}

[1] Bengtsson,H. 2017 vsnet-alert(T.Karo) $\sharp 20792$

[2] Hellier,C.2001 Cataclysmic Variable Stars Cambridge, Chapter 4

[3] Littlefair,S.P. et al. 2007 MNRAS 381,824

[4] Stellingwerf ,R.F.1978 ApJ 224,953

[5] Szkody ,P.et al. 2005 AJ 129,2386

[6] Uthas,H. et al. 2011 MNRAS 414,L85

[7] Uthas,H. 2011, PhD Thesis

\section{DISCUSSION}

VOJTECH SIMON: Are the bumps in the light curve of the outburst of OV Boo superhumps or some other features of the orbital modulation because their period is identical with the orbital period?

KENJI TANABE: As you mentioned, this period is quite identical to the previously obtained orbital one.So this outburst is different from the superoutburst of SU UMa or WZ Sge-type dwarf novae

\section{RYOKO ISHIOKA:}

1. What is the error of each observation?

2. Please explain me again the reason you concluded that this object is not SU UMa or WZ Sge. WZ Sge atars are known to show early superhumps at the early stage of superoutburst and the period is almost the same with the orbital period.

KENJI TANABE: Concerning the question 1,photometric precision at best is 0.015 magnitude.It varies sky condition. Concerning the 2 nd question,no subsequent outburst (so-called rebrightening) was yet observed.This is the reason why this outburst is different from the superoutburst of SU UMa or WZ Sge-type DN. Another reason is that the orbital period is shorter than the "period minimum" by ten minutes. 